\title{
Preparation of Chitosan Scaffolds for Tissue Engineering using Supercritical Fluid Technology
}

\author{
A.R.C. Duarte ${ }^{1,2}$ a , J.F. Mano ${ }^{1,2, b}$ and R.L. Reis ${ }^{1,2, c}$ \\ ${ }^{1} 3 \mathrm{~B}$ 's Research Group - Biomaterials, Biodegradables and Biomimetics, University of Minho, \\ Headquarters of the European Institute of Excellence on Tissue Engineering and Regenerative \\ Medicine, AvePark, 4806-909 Taipas, Guimarães, Portugal \\ ${ }^{2}$ IBB - Institute for Biotechnology and Bioengineering, PT Government Associated Laboratory, \\ Guimarães, Portugal \\ aaduarte@dep.uminho.pt, ${ }^{\mathrm{b}}$ jmano@dep.uminho.pt, ${ }^{\mathrm{c}}$ rgreis@dep.uminho.pt
}

Keywords: Supercritical fluids, Phase inversion, Biopolymers, Tissue engineering, Scaffolds, Chitosan

\begin{abstract}
The aim of this study was to evaluate the possibility of preparing chitosan porous matrixes using supercritical fluid technology. Supercritical immersion precipitation technique was used to prepare scaffolds of a natural biocompatible polymer, chitosan, for tissue engineering purposes. The physicochemical and biological properties of chitosan make it an excellent material for the preparation of drug delivery systems and for the development of new biomedical applications in many fields from skin to bone or cartilage.

Immersion precipitation experiments were carried out at different operational conditions in order to optimize the processing method. The effect of different organic solvents on the morphology of the scaffolds was assessed. Additionally, different parameters that influence the process were tested and the effect of the processing variables such as polymer concentration, temperature and pressure in the chitosan scaffold morphology, porosity and interconnectivity was evaluated by micro computed tomography. The preparation of a highly porous and interconnected structure of a natural material, chitosan, using a clean and environmentally friendly technology constitutes a new processing technology for the preparation of scaffolds for tissue engineering using these materials.
\end{abstract}

\section{Introduction}

The use of natural derived polymers for tissue engineering has been proposed in a number of different studies.[1] Chitin is widely found in shells of crustaceous such as crabs and shrimp and it is the second most abundant polymer after cellulose. Chitin forms strong inter- and intramolecular hydrogen bonds, which is difficultly broken by common solvents. Therefore its solubility in common solvents is rather constrained. For this reason, limited utilization of this natural resource has been reported. Up to date, the majority of uses of chitin are mainly related to chitosan, which is a cationic polymer derived from chitin comprising copolymers of $\beta(1 \rightarrow 4)$-glucosamine and $\mathrm{N}$ acetyl-d glucosamine. The physicochemical and biological properties of chitosan make it an excellent material for the preparation of drug delivery systems and for the development of new biomedical applications in many fields from skin to bone or cartilage.[1] Chitosan is usually processed from a diluted acetic acid solution, however this limits its processability.

Chitosan has been processed in several forms to be used in tissue engineering applications, namely, membranes, [2] particles[3], fibers and 3D fiber meshes[3, 4]. Chitosan may also be used as a drug delivery carrier and various studies have been reported in literature.[5,6] Chitosan sponges and scaffolds are also described in literature as deliver systems able to carry active agents or biomolecules and growth factors.[7] The preparation of these systems normally envolves freezedrying or lyophilizing a chitosan gel solution. $[8,9]$ Recently, it has been reported the use of supercritical fluid assisted phase inversion method for the preparation of chitosan membranes from a dilute acetic acid solution.[10] However, this process is time consuming and energy intensive. In 
this work we report the possibility of producing 3D chitosan scaffolds using organic solutions. Supercritical fluid technology presents great advantages over the conventional processes, due to the properties of the fluid phase, namely, high diffusivity. Although organic solvents are used they can be completely removed and a final product, dry and free of any residual solvent is obtained. Different solvents were tested in order to evaluate the effect of the solvent on the morphology of the materials obtained.

\section{Materials and Methods.}

Supercritical assisted phase-inversion process. The phase inversion experiments were carried out in an apparatus especially for this purpose[11] and schematically presented in figure 1.

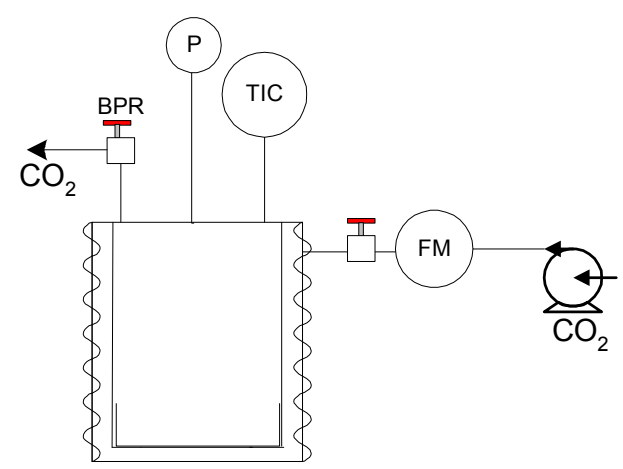

Fig. 1. Schematic diagram of the high pressure phase inversion apparatus $(\mathrm{P}-$ pressure transducer; $\mathrm{TIC}$ - temperature controller; FM - flowmeter; BPR - back pressure regulator).

Briefly, in each experiment a small amount (ca. $2 \mathrm{ml}$ ) of the polymer solution is loaded in a stainless steel cap with $2 \mathrm{~cm}$ diameter, which is placed inside the high pressure vessel. The vessel is heated in by means of an electric thin band heater (OGDEN) connected to a temperature controller, that maintains temperature within $\pm 1^{\circ} \mathrm{C}$ (TC). Carbon dioxide is pumped into the vessel using high pressure piston pump ( $\mathrm{P}-200 \mathrm{~A}$ Thar Technologies) until the operational pressure is attained. The pressure inside the vessel is measured with a pressure transducer $(\mathrm{P})$. The system was closed for 45 minutes to allow the occurrence of phase separation. Afterwards the system is flushed for another 45 minutes, with a stream of carbon dioxide at very low flow rate $(5 \mathrm{~g} / \mathrm{min})$, in order to ensure complete drying of the scaffolds. The flow is regulated by a flow meter (FM - Siemens, SITRANS FC MASS FLO MASS2100).

Scaffold characterization. Scanning Electron Microscopy - SEM: Samples of the scaffolds prepared were observed by a Leica Cambridge S360 Scanning Electron Microscope (SEM). The films were fixed by mutual conductive adhesive tape on aluminium stubs and covered with gold palladium using a sputter coater.

Micro-Computed Tomography $-\mu$-CT: The inner structure, porosity and interconnectivity were evaluated by micro-computerized tomography using a Scanco 20 equipment (Scanco Medicals, Switzerland) with penetrative X-rays of $40 \mathrm{keV}$. The X-ray scans were acquired in high-resolution mode $(39.39 \mu \mathrm{m})$. CT Analyser ${ }^{\circledR}$ (SkyScan, Belgium) was used to visualize the 2D X-ray sections images of the scaffolds.

Fourrier Transform Infra Red Spectroscopy - FTIR analysis was performed to the chitosan scaffolds prepared. Spectra were recorded at 32 scans with a resolution of $2 \mathrm{~cm}^{-1}$ (Shimadzu IRPrestige 21). 


\section{Results and Discussion.}

The experimental set up and the operating conditions used permited to process chitosan with a porous morphology. Supercritical assisted phase inversion process was used to produce 3D scaffolds of chitosan. Different organic solvents were tested in order to evaluate its effect on the morphology of the scaffolds obtained. In order to be able to compare the structures obtained the scaffolds were processed at the same operating conditions, $333 \mathrm{~K}$ and 150 bar. Fig. 2 represents the SEM images of the cross section of the scaffolds obtained.
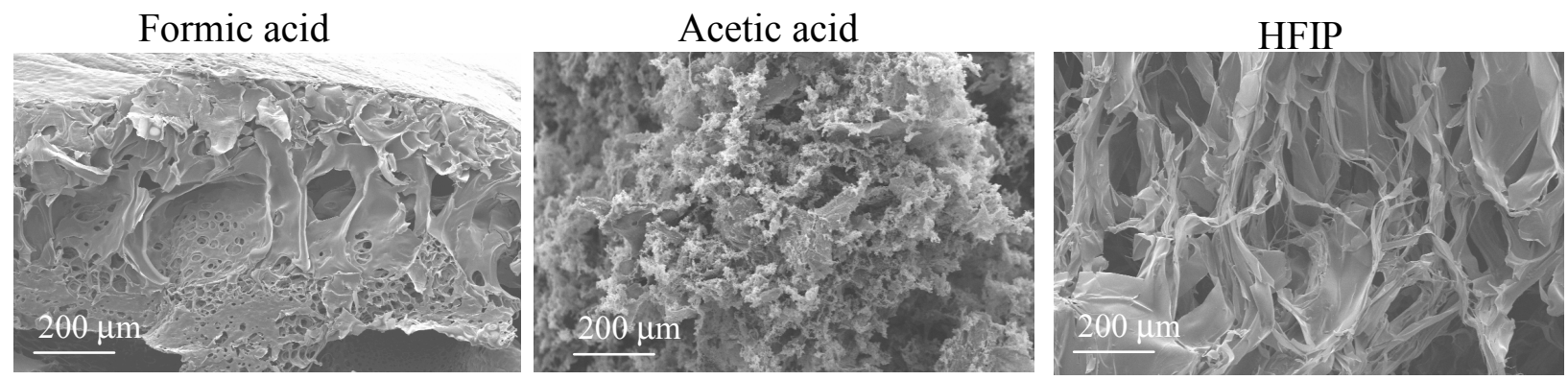

Fig. 2. SEM images of the scaffolds prepared from different organic solutions at $332 \mathrm{~K}$ and 150 bar.

From these images it is noticeable the strong dependence of the structure obtained with the solvent used. When chitosan was precipitated from HFIP or acetic acid solution a 3D structure was obtained. However, the scaffolds obtained from HFIP solution presented an upper compact layer, which was not observed in chitosan scaffolds precipitated from acetic acid. On the other hand, when a formic acid solution was processed a thin membrane with lower porosity was obtained. Micro CT allows the determination of the scaffold porosity which varies from $29 \%$, to $47 \%$ and $90 \%$ for the matrixes precipitated from formic acid, acetic acid and HFIP solutions, respectively. Additional information on the average pore size can also be taken from this analysis and from the SEM images. Scaffolds prepared from HFIP solution have larger pore diameters $(\sim 600 \mu \mathrm{m})$, when compared to the scaffolds prepared from acetic acid solution (mean pore size $\sim 110 \mu \mathrm{m}$ ) or from formic acid (mean pore size $\sim 60 \mu \mathrm{m}$ ).

The complexity of the mechanisms involved in the phase inversion process is associated with the interactions between the three components present in the system, solvent, non-solvent and polymer. A higher solubility between the organic solvent and the non-solvent will favour the process, which will cause phase separation and precipitation of the polymer with a porous structure.

In tissue engineering the preparation of a 3D matrix, highly porous and interconnected is crucial for the success of the implant. From three different organic solutions (formic acid, acetic acid and HFIP) we prepared very different materials, namely a dense chitosan membrane from formic acid, a macrovoid structure, with a dense layer on top from HFIP solution and a homogeneous scaffold with potential for tissue engineering applications from an acetic acid solution.

FTIR spectroscopy was performed to the materials processed from different solutions in order to evaluate the effect of the dissolution on chitosan. Figure 3 presents the FTIR spectra of the raw material and chitosan precipitated from HFIP, formic acid and acetic acid.

From the spectra obtained it is possible to conclude that there were no significant changes on chitosan structure when precipitated from HFIP or acetic acid solution. It gives us further information regarding the success of the solvent removal using supercritical carbon dioxide, as no traces of solvent are present on the spectra. 


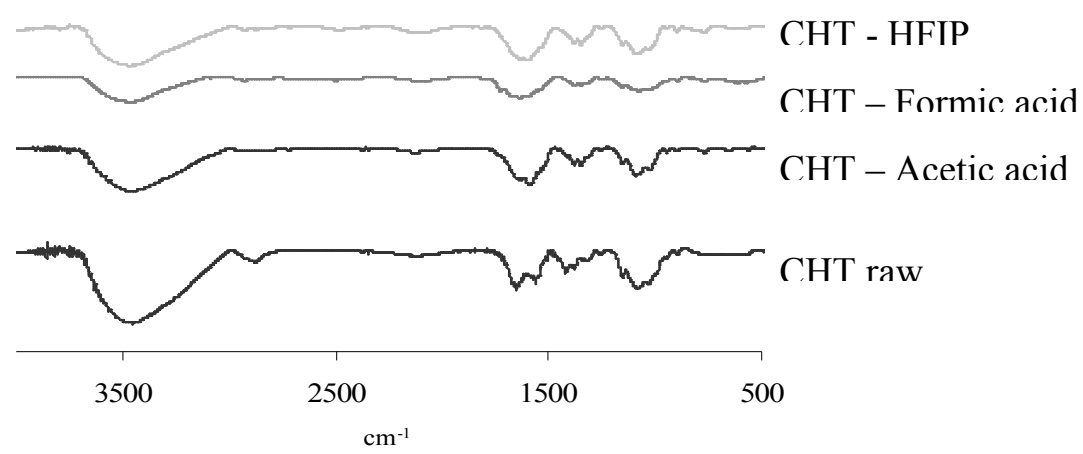

Fig. 3. FTIR spectra of the chitosan scaffolds precipitated from different organic solutions.

\section{Conclusions}

In this work we evaluate the feasibility of processing chitosan 3D scaffolds for tissue engineering applications using supercritical fluid technology. Supercritical assisted phase inversion was used to precipitate chitosan from HFIP, formic acid and acetic acid solutions. Different solvents were tested and we can conclude that the morphologies of the scaffolds obtained dependent greatly on the casting solvent used. The morphology of the scaffolds, its porosity and pore size varies with the type of casting solvent used and the interactions between the three components of the system (polymer + solvent + non-solvent) can help to explain the differences obtained.

\section{Acknowlegments}

Ana Rita C. Duarte is grateful for financial support from Fundação para a Ciência e Tecnologia (FCT) through the grant SFRH / BPD / 34994 / 2007. The support through the FCT project PTDC/QUI/68804/2006 is also acknowledged.

\section{References}

[1] J. F. Mano, G. A. Silva, H. S. Azevedo, P. B. Malafaya, R. A. Sousa, S. S. Silva, L. F. Boesel, J. M. Oliveira, T. C. Santos, A. P. Marques, N. M. Neves, R. L. Reis, Journal of the Royal Society Interface Vol. 4 (2007), p. 999-1030

[2] T. C. Santos, A. P. Marques, S. S. Silva, J. M. Oliveira, J. F. Mano, A. G. Castro, R. L. Reis, Journal of Biotechnology Vol. 132 (2007), p. 218-226

[3] M. Prabaharan, J. F. Mano, Drug Delivery Vol. 12 (2005), p. 41-57

[4] K. Tuzlakoglu, C. M. Alves, J. F. Mano, R. L. Reis, Macromolecular Bioscience Vol. 4 (2004), p. 811-819

[5] M. Prabaharan, M. A. Rodriguez-Perez, J. A. de Saja, J. F. Mano, Journal of Biomedical Materials Research Part B-Applied Biomaterials Vol. 81B (2007), p. 427-434

[6] J. Shi, N. M. Alves, J. F. Mano, Journal of Biomedical Materials Research Part B-Applied Biomaterials Vol. 84B (2008), p. 595-603

[7] O. R. M. Denkbas E.B., Journal of Bioactive Compatible Polymers Vol. 21 (2006), p. 351-368

[8] F. L. Mi, S. S. Shyu, Y. B. Wu, S. T. Lee, J. Y. Shyong, R. N. Huang, Biomaterials Vol. 22 (2001), p. 165-173

[9] K. Oungbho, B. W. Muller, International Journal of Pharmaceutics Vol. 156 (1997), p. 229-237

[10] M. Temtem, L. M. C. Silva, P. Z. Andrade, F. dos Santos, C. L. da Silva, J. M. S. Cabral, M. M. Abecasis, A. Aguiar-Ricardo, The Journal of Supercritical Fluids (2009) In Press

[11] A. R. C. Duarte, J. F. Mano, R. L. Reis, The Journal of Supercritical Fluids (2009) In Press 